\title{
Tipos de tarefas e estratégias de comunicação: investigando a validade de narrativas baseadas em tópicos e em figuras
}

\author{
Task type and Communication Strategies: Investigating \\ the Validity of Topic-Based and Picture-Based Narratives
}

\author{
Gicele Virgine Vieira Prebiana \\ Universidade Federal de Santa Catarina - UFSC \\ Rosane Silveira \\ Universidade Federal de Santa Catarina - UFSC
} Abstract

This study set up to investigate the validity of different oral tasks, namely topic-based and picture-based narratives, as data elicitation tasks. Our ultimate goal was to examine whether these two types of narratives would impact upon intermediate learners' L2 oral production, regarding their use of communication strategies (CS) during speech performance. Spearman correlational tests were run so as to check for ranking and frequency of the strategies used by participants in each narrative task. Results indicate that the type of task and its intrinsic characteristics may affect the use of CS in terms of frequency. Additionally, results show that the participants tended to experience fewer difficulties when performing the picturebased narrative, which led them to use a fewer number of strategies. On the contrary, the two topic-based narratives led participants to apply a greater number of CS.

Keywords

Communication Strategies, Task Type, Oral Narratives, EFL 


\section{Resumo}

Este estudo investiga a validade de duas tarefas orais diferentes - a narrativa baseada em tópicos e a narrativa baseada em figuras, como tarefas de elicitação de dados. Nosso objetivo principal foi o de examinar se esses dois tipos de tarefas causariam algum impacto na produção oral em língua estrangeira de aprendizes de nível intermediário, considerando seu uso de estratégias de comunicação (EC). Testes correlacionais Spearman foram rodados a fim de checar o ranqueamento e a freqüência das estratégias usadas pelos participantes em cada uma das narrativas. Os resultados indicam que o tipo de tarefa e suas características intrínsecas podem afetar o uso de EC em termos de frequiência. Além disso, os resultados também mostram que a narrativa baseada em figuras parece ter causado menos problemas para os aprendizes, que, por sua vez, utilizaram um número menor de EC. Já as narrativas baseadas em tópicos levaram os participantes a utilizar um maior número de estratégias.

\section{Palavras-chave}

Estratégias de Comunicação, Tipo de Tarefa, Narrativas Orais, EFL 


\section{Introdução}

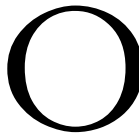

presente estudo teve como objetivo investigar se diferentes tipos de narrativas - as baseadas em tópicos e as baseadas em figuras determinam a freqüência e o tipo de Estratégias de Comunicação (EC) utilizadas por aprendizes de nível intermediário de inglês como língua estrangeira. Neste sentido, o presente estudo encontra suporte em duas linhas de pesquisa distintas - uma que discute o uso de tarefas do ponto de vista do ensino/aprendizagem e da testagem e sua influência sobre o desempenho oral de aprendizes de língua estrangeira, e outra que tem se concentrado em determinar como esses aprendizes lidam com problemas comunicativos.

Apesar de muitos estudos terem examinado os efeitos de várias tarefas e do uso de EC na produção oral (POULISSE \& SCHILS, 1989; CHEN, 1990; PARIBAKHT, 1985; YARMOHAMMADI \& SEIF, 1992; VARADI, 1983; TARONE, 1980; FAERCH \& KASPER, 1983; DÖRNYEI \& KORMOS, 1998; FOSTER \& SKEHAN, 1996; SKEHAN \& FOSTER, 1999), parece ainda haver dúvida se o uso de tarefas específicas desencadeia ou proporciona o uso de determinadas EC. Além disso, há ainda a necessidade de se avaliar a competência estratégica dos aprendizes de língua estrangeira em diferentes eventos comunicativos (FAERCH \& KASPER, 1983). Sendo assim, o presente estudo tem a finalidade de fornecer dados que enriqueçam a literatura da área, investigando o uso de EC por aprendizes de língua estrangeira em três tarefas diferentes: duas narrativas baseadas em tópicos e uma narrativa baseada em figuras. A fim de alcançar tal objetivo, este estudo procurou responder às seguintes perguntas de pesquisa:

1. Há um padrão consistente no ranqueamento do uso de estratégias comunicativas para diferentes tipos de tarefas?

2. Há um padrão consistente no uso de estratégias comunicativas para cada participante nas três tarefas? 
Este artigo está organizado em cinco principais seções. Na primeira, nós oferecemos uma ampla visão das múltiplas perspectivas e linhas de pesquisa na área de tarefas. Na segunda seção, relevantes pesquisas sobre EC são revisadas. A terceira seção apresenta a descrição do método, dos participantes e dos procedimentos de coleta e análise de dados. Finalmente, a quarta seção, reporta os resultados e a discussão geral deles. Algumas considerações finais a respeito das limitações do estudo e sugestões para futuras pesquisas são apresentadas na quinta seção.

\section{Múltiplas perspectivas sobre tarefas}

Tarefaé um conceito multifacetado que, devido às suas várias interpretações, pode ser mal interpretado e mal aplicado (BYGATE, SKEHAN \& SWAIN, 2001). Como explicado por Skehan (2003), três grupos de pessoas estão interessados em tarefas: (i) pesquisadores, (ii) elaboradores de testes e (iii) professores. Primeiramente, no caso dos pesquisadores, uma tarefa é geralmente vista como uma atividade que irá fornecer dados para futuras análises. Em segundo lugar, quando elaboradores de testes se referem a tarefas, eles estão interessados em examinar a confiabilidade e validade dos métodos utilizados para elicitação de dados e em determinar até que ponto a estrutura da tarefa pode influenciar o desempenho dos que as realizam (BACHMAN \& PALMER, 1996; BYGATE, SKEHAN \& SWAIN, 2001). Finalmente, professores tendem a interpretar tarefas como sendo atividades de sala de aula que possuem um objetivo pedagógico definido. Em outras palavras, a maior preocupação dos professores em relação ao uso de tarefas de cunho pedagógico em sala de aula é com questões instrucionais (SKEHAN, 2003; BYGATE, SKEHAN \& SWAIN, 2001).

Em geral, pesquisas sobre tarefas têm sido conduzidas com base em três perspectivas principais: a psicolingüística, a sociocultural e a cognitiva (SKEHAN, 2003). Na perspectiva psicolinguiística, o foco é dado aos tipos de tarefas que permitem negociação de significado (LONG, 1983 em SKEHAN, 2003) e reformulação do enunciado (recast) (LONG, INAGAKI \& ORTEGA, 1998; GASS, 2002 em SKEHAN, 2003) como formas de promover o aprendizado da língua por meio de interação quando falhas no processo comunicativo são iminentes. Por outro lado, a perspectiva sociocultural enfatiza o uso de tarefas como oportunidades para aprendizes interagir e co-construir significado colaborativamente. Nessa perspectiva, a negociação de significado não é 
essencial, devido ao fato de que nenhuma lacuna na comunicação é necessária para a interação acontecer. Ainda, segundo Skehan (2003), diferentes aspectos das tarefas têm sido objetos de estudo na perspectiva cognitiva. Esses aspectos envolvem a alocação de recursos atencionais pelos aprendizes de língua estrangeira durante a realização das tarefas e a influência das características intrínsecas e condições de desempenho das tarefas sobre a produção oral.

No tocante ao uso de tarefas de cunho pedagógico, Ellis (2003) propõe que é necessário identificar alguns critérios a fim de levar em conta as similaridades entre as tarefas. Sendo assim, Ellis afirma que uma tarefa deve (i) representar um plano para o professor, no qual ele encontre suporte para a sua prática; (ii) ser, primariamente, uma atividade que envolva significado; (iii) ter semelhança com atividades reais (de fora da sala de aula); (iv) envolver as quatro habilidades lingüísticas; (v) estimular processos cognitivos e (vi) produzir um resultado.

Seguindo a perspectiva da área de testagem, por outro lado, Bachman e Palmer (1996), além de definir uma tarefa como "uma atividade que envolve o uso da língua por indivíduos, a fim de alcançar um determinado objetivo em uma determinada situação" (p. 44, tradução das autoras), enfatizam a necessidade de entender que as características intrínsecas das tarefas podem afetar a performance dos aprendizes em uma determinada situação de testagem. Portanto, Bachman e Palmer alegam que o design da tarefa é o único fator sob controle dos elaboradores de testes e/ou dos professores. É nessa perspectiva de que as tarefas podem ser utilizadas como método de elicitação de dados que o presente estudo se apóia.

A importância de investigar os efeitos das características intrínsecas das tarefas também é enfatizada por Ellis (2003). Como explicado pelo autor, o grande desafio em examinar tais variáveis é que elas podem interagir com outras, tornando difícil a identificação de qual ou quais variáveis estão realmente causando um determinado efeito. As variáveis que precisam ser levadas em consideração quando do desenvolvimento das tarefas, tanto para fins pedagógicos, quanto de pesquisa ou de testagem, dizem respeito (i) ao tipo de insumo oferecido aos aprendizes - se ele será oral ou visual e se os aprendizes terão acesso ao material durante a realização da tarefa, (ii) ao número de elementos que os aprendizes precisarão manipular para completar a tarefa e (iii) ao tópico dessa tarefa - se os aprendizes estão familiarizados com esse tópico e se alguns tópicos demandam mais esforço cognitivo do que outros (ELLIS, 2003). 
Em um de seus trabalhos, Robinson (2001) afirma que a complexidade e a dificuldade da tarefa também podem interferir no resultado obtido. Robinson distingue entre complexidade e dificuldade, explicando que, enquanto a primeira está relacionada à estrutura da tarefa e suas características intrínsecas, sendo então passível de manipulação pelos professores, a última é estabelecida de acordo com o grau de afetividade dos aprendizes em relação à tarefa e ao processo de ensino/ aprendizagem. Em outras palavras, a complexidade da tarefa refere-se às demandas cognitivas da tarefa, enquanto a dificuldade da tarefa está ligada a diferenças nos estilo de aprendizagem, aptidão e motivação dos aprendizes.

Foster e Skehan (1996) investigaram se três tarefas diferentes influenciariam a produção oral dos aprendizes em termos de fluência, acurácia e complexidade. Eles usaram uma tarefa de troca de informação pessoal (mais estruturada, com um único resultado possível e informação familiar), uma narrativa (menos estruturada, mas com suporte visual) e, por fim, uma tarefa de tomada de decisão (não estruturada, com mais de um resultado possível e com informação não familiar). Os resultados indicaram desempenhos diferentes de acordo com o tipo de tarefa realizada. Os maiores índices de acurácia foram encontrados na tarefa de troca de informação pessoal e na de tomada de decisão. A narrativa e a tomada de decisão produziram discurso menos fluente do que a outra tarefa, enquanto a tarefa de troca de informação pessoal gerou um discurso menos complexo em comparação às outras tarefas.

Similarmente, Skehan e Foster (1999) reportaram uma interação de fatores que afetam o desempenho oral em língua estrangeira - a estrutura da tarefa e as condições de processamento. Os pesquisadores examinaram a fluência oral, a acurácia e a complexidade geradas na realização de uma narrativa relativamente estruturada e uma outra relativamente não estruturada, desempenhadas sob quatro diferentes condições de processamento: (1) assistir e contar simultaneamente, (2) assistir e contar simultaneamente após ouvir a estória de um vídeo, (3) assistir primeiro, então assistir e contar simultaneamente e (4) assistir primeiro e depois contar. Em geral, os resultados sugerem que

a fluência foi principalmente afetada pelo grau da estrutura da tarefa; a complexidade foi principalmente afetada pelas condições sob as quais as tarefas foram realizadas...e a acurácia foi afetada pela interação da estrutura da tarefa e pela oportunidade de engajar em algum estágio de preparação para a tarefa. (SKEHAN \& FOSTER, 1999, p. 113-114, tradução das autoras). 
Em suma, a breve revisão de literatura aqui apresentada parece indicar que sempre que o termo tarefa é utilizado, faz-se necessário posicioná-lo dentro de um referencial teórico a fim de evitar mal-entendidos e favorecer a constante avaliação dos possíveis efeitos das características das tarefas sobre os resultados da realização dessas tarefas.

\section{Estratégias de Comunicação}

O debate sobre o uso de estratégias de comunicação (EC) tem uma longa tradição na agenda de pesquisa de aquisição/aprendizado de uma língua estrangeira e tem contribuído para o esclarecimento de uma série de variáveis relativas ao uso de estratégias como o escopo do construto, os critérios de definição para identificar uma estratégia e as taxonomias utilizadas para a classificação dessas estratégias.

O termo estratégias de comunicação apareceu primeiramente num estudo de Selinker, em 1972, como mecanismos aplicados por aprendizes de uma língua estrangeira com a finalidade de se comunicar com falantes nativos dessa língua. Desde então, o termo tem sido entendido como dispositivos aplicados de forma consciente pelos aprendizes para resolução de problemas comunicativos. Tais dispositivos são normalmente utilizados quando os aprendizes sentem uma lacuna entre o conhecimento lingüístico necessário para comunicar a mensagem desejada na língua-alvo e suas reais capacidades comunicativas (VARADI, 1983; FAERCH \& KASPER, 1983; DÖRNYEI \& SCOTT, 1997; DÖRNYEI \& KORMOS, 1998). Essa lacuna refere-se, então, a déficits que podem estar relacionados não só ao conhecimento lingüístico incompleto dos aprendizes, mas também a aspectos da sua própria performance, da performance dos seus interlocutores ou a déficits causados pela restrição do tempo em que a fala deve ser produzida (DÖRNYEI \& KORMOS, 1998).

As EC também têm sido investigadas do ponto de vista interacional, ou seja, segundo Tarone (1980), a língua consiste de eventos interacionais que levam falantes e interlocutores a uma troca constante de informação a fim de promover comunicação. Essa troca, de acordo com Tarone, é essencial para que falantes e interlocutores compartilhem significados. No entanto, uma vez que eles não são compartilhados, faz-se necessário o uso de estratégias de comunicação para que ocorra uma comunicação eficiente. 
O fato do emprego das EC por falantes e interlocutores estar intimamente ligado a eventos interacionais na proposta de Tarone (1980) causou algumas críticas. De acordo com Faerch e Kasper (1983), a interação tomada como base para o emprego de EC, como proposto por Tarone, não é uma condição necessária para tanto. Os autores alegam que os aprendizes (falantes e/ou interlocutores) podem preferir solucionar seus problemas comunicativos sem apelar para os seus interlocutores. Faerch e Kasper (1983) propõem, por outro lado, uma abordagem psicolingüística, na qual o uso de EC está relacionado aos processos cognitivos de produção da fala. Esses autores sugerem que, para alcançar seu objetivo comunicativo, os aprendizes realizam uma série de processos cognitivos de planejamento e execução dos objetivos. Sendo assim, se eles não conseguem realizar tais processos e, conseqüentemente, o plano comunicativo original, precisam recorrer ao uso das EC.

A maioria das definições para o construto estratégias de comunicação relaciona-o à resolução de problemas comunicativos (FAERCH \& KASPER, 1983; DÖRNYEI \& KORMOS, 1998; DÖRNYEI \& SCOTT, 1997). Todavia, tal critério tem sido amplamente criticado por Bialystok (1990). Essa autora alega haver duas grandes implicações a respeito do critério problematicidade. Primeiramente, segundo Bialystok, parece não haver uma explicação clara de como os aprendizes de língua estrangeira separam o uso estratégico da língua do uso da língua para a comunicação cotidiana, quando nenhum problema comunicativo é detectado. Em segundo lugar, as EC não são apenas empregadas por aprendizes de língua estrangeira. Elas podem ser utilizadas por falantes nativos que visam assegurar o sucesso das suas jornadas comunicativas.

Pesquisas sobre EC não têm apenas se preocupado em definir o construto. Há também esforços para determinar taxonomias que permitam a identificação e classificação dessas estratégias. As taxonomias mais citadas na literatura da área são as desenvolvidas por Tarone (1980), Faerch e Kasper (1983), Bialystok (1990), o grupo Nijmegen (KELLERMAN, 1991; KELLERMAN, BONGAERTS \& POULISSE, 1987, entre outros) e Dörnyei e Kormos (1998).

Um dos maiores problemas em relação às taxonomias é o fato de que a maioria delas classifica as EC baseando-se em suas características superficiais, não revelando realmente os processos cognitivos que levam ao uso de tais estratégias. Sendo assim, a crítica principal do Grupo Nijmegen está relacionada à falta de plausibilidade psicológica dessas taxonomias, ou seja, as diferentes 
categorias de EC propostas parecem não representar processos cognitivos diferentes e, portanto, não deveriam ser classificadas diferentemente.

Contudo, a fim de solucionar os problemas apontados pelo Grupo Nijmegen, Dörnyei e Kormos (1998) propõem uma taxonomia para classificação das EC que as relaciona com os processos cognitivos realizados pelos falantes ao produzirem sua fala. Para tanto, Dörnyei e Kormos utilizam o modelo de fala proposto por Levelt (1989), o qual divide a produção oral em três estágios: conceitualização, formulação e articulação. As EC, segundo Dörnyei e Kormos, representam, de certa forma, os processos cognitivos envolvidos nesses três estágios, uma vez que, ao utilizar uma EC, o falante precisará replanejar seus conceitos, reformular as características semânticas, gramaticais e fonológicas da sua mensagem e, por fim, monitorar novamente o resultado desses processos a fim de verificar se sua intenção comunicativa foi realizada.

Tomemos como exemplo o uso da estratégia de reestruturação (restructuring). Ao aplicar essa EC, o falante, em certo momento da sua fala, precisará reestruturar sua mensagem pré-verbal, isto é, aquilo que deseja comunicar, suas idéias e seus conceitos. Ao fazer isso, ele precisa replanejar sua fala e selecionar os itens lexicais adequados para transmissão da mensagem oral pretendida. No exemplo a seguir, produzido por um dos participantes desta pesquisa, podemos verificar que tais processos cognitivos (reconceitualização, replanejamento, seleção lexical) estão envolvidos na reestruturação da mensagem que o falante queria comunicar:

I: I started to:... when I finished my law school I only start to study English and Portuguese

No tocante aos estudos empíricos, a maioria das pesquisas sobre EC tem se concentrado em estudar (1) a influência dos diferentes tipos de tarefas e níveis de proficiência no uso de EC (POULISSE \& SCHILS, 1989; CHEN, 1990; PARIBAKHT, 1985, e YARMOHAMMADI \& SEIF, 1992), (2) o uso de EC em sala de aula (FLYMAN, 1997; RODRIGUES, 1999), e (3) o ensino das EC aos falantes de língua estrangeira (treinamento estratégico) (MANCHÓN, 2000).

Em resumo, a literatura na área de EC tem demonstrado uma falta de consenso em relação à melhor forma de definir, conceitualizar e classificar as estratégias de comunicação. Claramente, mais pesquisas são necessárias a fim de solucionar esses aspectos. 


\section{Método}

\subsection{Participantes}

Os participantes do presente estudo foram 10 aprendizes de inglês como língua estrangeira do nível intermediário do curso extracurricular da Universidade Regional de Blumenau, com nenhuma experiência em um país de língua inglesa. Esses aprendizes também participaram de outro estudo conduzido por Prebianca (2004) e foram selecionados devido ao fato de que, naquele estudo em particular, eles aplicaram um maior número de EC e apresentaram um repertório de estratégias mais amplo em relação aos aprendizes dos níveis pré-intermediário e avançado.

\subsection{Coleta e Análise de dados}

Visando alcançar os objetivos deste estudo, os dados analisados fazem parte de um conjunto de dados coletados para o estudo de Prebianca (2004). As sessões de coleta de dados foram conduzidas no período correspondente a um semestre acadêmico da Universidade Regional de Blumenau. A produção oral dos aprendizes foi elicitada através de três narrativas - duas baseadas em tópicos e uma baseada em figuras. Na primeira narrativa baseada em tópico, os aprendizes contaram um fato das suas vidas que os tinha feito felizes. $\mathrm{Na}$ segunda narrativa do mesmo tipo, eles contaram a estória de um filme ao qual haviam assistido. Na narrativa baseada em figuras, foi-lhes mostrada uma sequiência de figuras de um gibi sem nenhuma linguagem escrita disponível, e foilhes pedido que narrassem o que estava acontecendo na estória. Os aprendizes tiveram 5 minutos no máximo para a realização de cada tarefa. Não foi permitido que eles interagissem com o pesquisador, nem que perguntassem sobre nenhum tipo de vocabulário desconhecido. Esses procedimentos foram adotados a fim de evitar o uso de algumas EC em detrimento de outras.

A fala dos participantes elicitada por meio dessas narrativas foi analisada de acordo com a taxonomia proposta por Dörnyei e Kormos (1998) para identificação e classificação das estratégias de comunicação. As definições e exemplos para as $31 \mathrm{EC}$ adotadas no presente estudo podem ser encontradas em Prebianca (2004).

Os dados das narrativas foram utilizados para responder a duas perguntas de pesquisa. A primeira delas indaga se existe um padrão consistente no uso de 
EC para as três tarefas narrativas utilizadas para elicitar a produção oral dos participantes. Para responder a essa pergunta, foram utilizadas as freqüências totais, por tarefa, das 31 EC investigadas por Prebianca (2004). O foco dessa análise é a frequiência total e o ranqueamento das $31 \mathrm{EC}$ por tarefa e, para tanto, os dados foram tabulados por tipo de EC para cada tarefa. A análise desses dados consistiu em verificar se o ranqueamento (com base na frequiência total) das 31 EC era semelhante para as três tarefas, valendo-se do teste correlacional não paramétrico Spearman, uma vez que as variáveis aqui utilizadas são do tipo ordinal.

A segunda pergunta que norteia este trabalho investiga a existência de um padrão consistente no uso de EC entre os 10 participantes para as três tarefas, utilizando as 31 EC do trabalho de Prebianca (2004). O foco dessa análise é a freqüência das 31 EC por participante, o que permite discutir diferenças individuais entre eles. Para essa análise, os dados foram tabulados levando em consideração as frequiências de EC para cada participante e para cada tarefa. Novamente foram rodados testes correlacionais Spearman, tendo em vista o caráter não paramétrico dos dados.

Nesta seção foram apresentadas informações sobre os participantes e o método utilizado para coletar e analisar os dados. A seção seguinte apresentará a discussão dos resultados.

\section{Resultados e Discussão}

Os resultados serão discutidos a seguir, tendo como base as duas perguntas de pesquisa que nortearam este trabalho. Passemos à discussão dos resultados para a primeira pergunta:

$\checkmark \quad$ Pergunta de Pesquisa 1: Há um padrão consistente no ranqueamento do uso de estratégias comunicativas para diferentes tipos de tarefas?

Os resultados apresentados na Tabela 1 contêm as freqüências, porcentagens e ranqueamentos (quanto maior o número, mais freqüente o uso da respectiva EC por tarefa) das $31 \mathrm{EC}$, para as três tarefas narrativas desempenhadas pelos participantes. Uma constatação imediata é a de que o número de EC utilizadas com as narrativas baseadas em tópicos (tarefas I e II) é quase duas vezes maior do que com a narrativa baseada em figuras (tarefa III). Esse resultado parece estar relacionado ao fato de que a narrativa baseada em figuras é uma tarefa que 
oferece suporte visual para o participante. Segundo Robinson (2001), esse tipo de tarefa demanda menos atenção e, conseqüentemente, libera recursos de memória que podem ser aplicados com outros aspectos da tarefa.

No caso dos participantes deste estudo, talvez eles tenham se valido do suporte contextual para dar conta da exigência cognitiva criada ao produzir fala em tempo real. As figuras podem ter auxiliado os participantes a trazer para a memória de trabalho os itens lexicais apropriados e estruturas lingüísticas necessárias para realizar a tarefa, facilitando, assim, o desempenho e prevenindo problemas de comunicação, o que pode ter levado os participantes a recorrer às EC com menor freqüência. Esses resultados corroboram os de Robinson (1995, em ELLIS, 2003), os quais sugerem que as narrativas produzidas quando os aprendizes tinham acesso a figuras criaram amostras de fala mais fluentes do que as narrativas realizadas sem suporte visual.

\section{TABELA 1}

Freqüência, porcentagem e ranqueamento do uso de EC

para as três tarefas narrativas

\begin{tabular}{|c|c|c|c|c|c|c|c|c|c|c|}
\hline Tipos de EC & T-I & $\%$ & Rank. & T-II & $\%$ & Rank. & T-III & $\%$ & Rank. & Total \\
\hline $\begin{array}{l}\text { 1. Abandono da } \\
\text { mensagem }\end{array}$ & 10 & 0,81 & 17,0 & 11 & 0,90 & 17,0 & 5 & 0,63 & 17,5 & 26 \\
\hline $\begin{array}{l}\text { 2. Redução da } \\
\text { mensagem }\end{array}$ & 3 & 0,24 & 11,5 & 2 & 0,16 & 7,5 & 1 & 2,78 & 8,0 & 0 \\
\hline $\begin{array}{l}\text { 3. } \begin{array}{l}\text { Substituição da } \\
\text { mensagem }\end{array}\end{array}$ & 2 & 0,16 & 9,5 & & 0,00 & 3,0 & & 0,00 & 2,5 & 2 \\
\hline $\begin{array}{l}\text { 4. Mudança de } \\
\text { código }\end{array}$ & 16 & 1,30 & 20,0 & 21 & 1,73 & 20,0 & 23 & 2,92 & 24,5 & 60 \\
\hline 5. Aproximação & 3 & 0,24 & 11,5 & 8 & 0,66 & 16,0 & 18 & 2,28 & 22,0 & 29 \\
\hline $\begin{array}{l}\text { 6. Palavras de } \\
\text { uso geral }\end{array}$ & 11 & 0,90 & 18,5 & 0 & 0,00 & 3,0 & 0 & 0,00 & 2,5 & 11 \\
\hline 7. Omissão completa & 7 & 0,57 & 15,0 & 13 & 1,07 & 19,0 & 7 & 0,89 & 20,0 & 27 \\
\hline 8. Estrangeirismo & 9 & 0,73 & 16,0 & 6 & 0,49 & 14,0 & 2 & 0,25 & 12,0 & 17 \\
\hline $\begin{array}{l}\text { 9. Criação de palavra } \\
\text { gramatical }\end{array}$ & 1 & 0,08 & 6,5 & 4 & 0,33 & 10,0 & 1 & 0,13 & 8,0 & 6 \\
\hline 10. Tradução literal & 1 & 0,08 & 6,5 & 5 & 0,41 & 12,0 & 1 & 0,13 & 8,0 & 7 \\
\hline 11. Circunlocução & 1 & 0,08 & 6,5 & 0 & 0,00 & 3,0 & 1 & 0,13 & 8,0 & 2 \\
\hline $\begin{array}{l}\text { 12. Criação de palavra } \\
\text { semântica }\end{array}$ & 0 & & 2 & 0 & & 3,0 & 0 & 0,00 & 2,5 & 0 \\
\hline 13. Reestruturação & 11 & 0,90 & 18,5 & 7 & 0,58 & 15,0 & 3 & 0,38 & 13,5 & 21 \\
\hline
\end{tabular}


(Continuação...)

14. Apelo direto

15. Apelo indireto

16. Generalização excessiva

17. Transferência

18. Redução gramatical

19. Recuperação fonológica

20. Substituição fonológica

21. Redução fonológica

22. Pausas preenchidas

23. Pausas não preenchidas

24. Umming/erring

25. Prolongamento de som

26. Auto-repetição

27. Retificação de erro

28. Retificação de apropriabilidade

29. Retificação distinta

30. Retificação com reformulação

31. Auto-verificação de acurácia

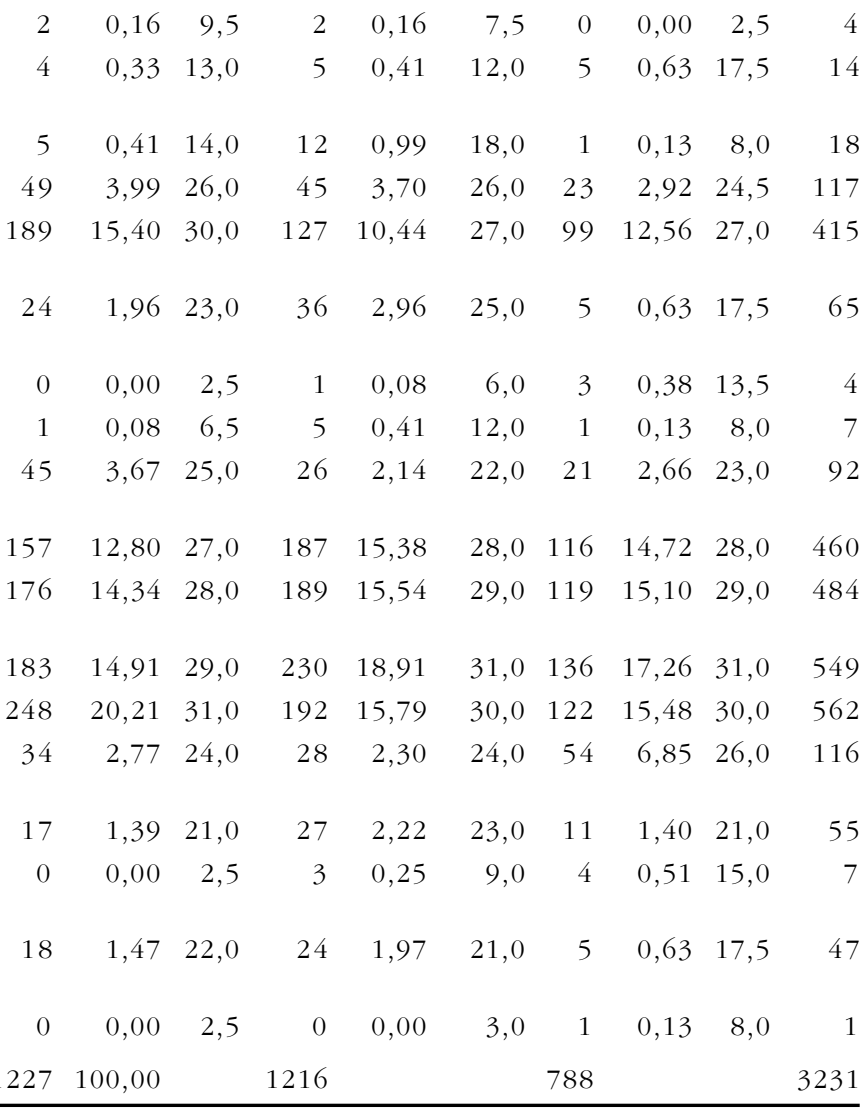

Total

Percentagens calculadas a partir dos totais por tarefas.

Cabe destacar que, em um grupo de $5 \mathrm{EC}$, essas estratégias aparecem como as mais frequientes nas três tarefas, respondendo por $76.44 \%$ do total de EC utilizadas: redução gramatical, pausas não preenchidas, umming/erring, ${ }^{1}$ prolongamento de som e auto-repetição. Por outro lado, outras $6 \mathrm{EC}$ aparecem como as menos freqüentes, representando menos de $1 \%$ do total de EC: criação de palavra semântica, auto-verificação de acurácia, substituição da mensagem, circunlocução, substituição fonológica e apelo direto. Note-se que esta última, apelo direto, não deveria ter sido utilizada, visto que as instruções dadas aos participantes assinalavam que eles não deveriam interagir com a professorapesquisadora; entretanto, essa EC foi utilizada duas vezes nas tarefas I e II, respectivamente. 
Os dados da Tabela 1 também indicam que determinadas EC são mais frequientes em algumas das tarefas. Por exemplo, pode-se observar que as EC palavras de uso geral, reestruturação e pausas preenchidas são mais freqüentes com a Tarefa I (narrativa baseada no tópico "memórias de um momento feliz"), ao passo que as EC criação de palavra gramatical, tradução literal, generalização excessiva, redução fonológica e retificação de apropriabilidade são mais freqüentes na tarefa II (narrativa baseada no tópico "relato de filme"). Já para a tarefa III (narrativa baseada em figuras), destacam-se o uso das EC mudança de código, aproximação e retificação de erro.

Devido ao número reduzido de dados do presente estudo, é difícil interpretar exatamente como o tipo de tarefa influencia o uso de EC. Entretanto, é possível observar que, no caso da Tarefa III, as EC que foram utilizadas com maior freqüência parecem estar relacionadas com o fato de que os participantes tinham que seguir o curso da estória representada na seqüência de figuras e isso não lhes permitiu fugir muito do contexto. Sendo assim, se não sabiam como dizer, por exemplo, bull ("touro"), tinham que usar uma palavra aproximada, ou usar o português. $\mathrm{O}$ fato de ter que contar exatamente o que viam pode ter restringido de alguma forma o uso de outras EC. Ainda para a Tarefa III, a alta freqüência da EC retificação do erro pode ser explicada da mesma forma: levados a contar o que viam nas figuras, precisaram se corrigir mais vezes a fim de ser o mais fiel possível ao insumo. Já com relação às Tarefas I e II, as EC que foram utilizadas com mais freqüência parecem ser utilizadas em situações mais amplas, ou seja, não restritas a um contexto. Para essas tarefas, os contextos eram memórias de vida e de um filme, e esses tópicos davam mais liberdade aos participantes de usar sua criatividade ou viajar na sua imaginação, pois não precisavam necessariamente contar algo real.

Em linhas gerais, os resultados mostram que os padrões de uso de EC são bastante semelhantes, tanto para as duas tarefas narrativas baseadas em tópicos quanto para a narrativa baseada em figuras. Para buscar suporte para essa interpretação, foram rodadas três correlações Spearman, buscando verificar se o ranqueamento da frequiência de uso das 31 EC era semelhante nas três tarefas. Como mostram os resultados da Tabela 2, há uma correlação forte e estatisticamente significativa ( $p<.0001)$ entre o ranqueamento das EC para as três tarefas, indicando que o tipo de tarefa não é um fator preponderante para 
a escolha do tipo de EC. Assim, independentemente do tipo de tarefa, os participantes tendem a adotar padrões semelhantes de uso de estratégias para minimizar problemas para se comunicar em língua inglesa.

TABELA 2

Testes correlacionais Spearman com as três tarefas narrativas

\begin{tabular}{lccc}
\hline \multirow{2}{*}{ Coeficiente } & Tarefa I - Tarefa II & Tarefa I - Tarefa III & Tarefa II - Tarefa III \\
\cline { 2 - 4 } Correlacional & .889 & .794 & .903 \\
Valor de p & .0001 & .0001 & .0001 \\
\hline
\end{tabular}

Passaremos agora à discussão dos resultados da segunda pergunta de pesquisa:

$\checkmark \quad$ Pergunta de Pesquisa 2: Há um padrão consistente no uso de estratégias comunicativas para cada participante nas três tarefas?

A análise dos dados para responder à primeira pergunta de pesquisa revelou a existência de um padrão consistente no uso de EC para as três tarefas desempenhadas pelos participantes. Tal análise, entretanto, desconsiderou a dimensão dos dados por participantes, que consiste no foco principal da segunda pergunta de pesquisa. Ao analisar a freqüência e o ranqueamento das EC por participantes, esperamos encontrar suporte para os resultados obtidos para a primeira pergunta de pesquisa, bem como vislumbrar resultados que possam ser atribuídos a diferenças individuais entre os 10 participantes. Os dados utilizados para essa análise se encontram no Apêndice (Tabela 5) e, resumidamente, na Tabela 4.

A Tabela 3 apresenta as intercorrelações, utilizando o teste Spearman, para o ranqueamento das EC para cada participante nas tarefas narrativas. Para todos os sujeitos, foram obtidas correlações significativas $(\mathrm{p}<.01)$, sendo a maioria dessas correlações de moderada a alta. Esses resultados confirmam os resultados obtidos para a primeira pergunta de pesquisa, visto que é possível afirmar que, de modo geral, cada um dos participantes apresentou padrões consistentes no uso de EC para as três tarefas. Isso significa que, embora as tarefas I e II tenham suscitado uma quantidade maior de EC do que a tarefa III, o ranqueamento das EC para cada uma das tarefas foi bastante semelhante para os 10 participantes. 
TABELA 3

Intercorrelações para cada participante nas três tarefas

(teste de Spearman)

\begin{tabular}{lrrrrrrrrrr}
\hline Participantes & 1 & 2 & 3 & 4 & 5 & 6 & 7 & 8 & 9 & 10 \\
\hline Tarefa I - Tarefa II & .851 & .667 & .485 & .797 & .743 & .776 & .680 & .772 & .775 & .846 \\
Tarefa I - Tarefa III & .677 & .672 & .682 & .633 & .666 & .642 & .439 & .514 & .840 & .820 \\
Tarefa II - Tarefa III & .686 & .754 & .514 & .515 & .914 & .689 & .681 & .646 & .774 & .683 \\
\hline
\end{tabular}

Todas as correlações são significativas com $\mathrm{p}<.01$.

Dando continuidade à análise dos dados por participantes, a Tabela 4 mostra que alguns deles recorreram a uma variedade maior de EC em uma ou mais tarefas. Por exemplo, na tarefa I, o participante 4 foi responsável por quase $16 \%$ das EC, enquanto o participante 3 respondeu por apenas $6.7 \%$. Situação semelhante ocorreu na tarefa II, na qual o participante 5 utilizou quase $15 \%$ das EC, enquanto o participante 3 utilizou apenas $6 \%$ das EC. Por fim, na tarefa III, os participantes 2 e 4 foram responsáveis por cerca de $13 \%$ das EC utilizadas, ao passo que o participante 3 foi novamente o que menos utilizou EC (4.8\%). Esses resultados evidenciam que o uso de EC é influenciado por diferenças individuais. Os participantes tendem a utilizar uma quantidade diferente de estratégias comunicativas para desempenhar tarefas narrativas, embora o tipo de estratégias de comunicação utilizado seja bastante semelhante.

TABELA 4

Freqüência de uso de EC por participante para as três tarefas

\begin{tabular}{llllllcllllll}
\hline & \multicolumn{10}{c}{ Tarefa I } \\
Partic. & 1 & 2 & 3 & 4 & 5 & 6 & 7 & 8 & 9 & 10 & Total \\
Freq. & 96 & 96 & 82 & 196 & 122 & 101 & 119 & 122 & 146 & 147 & 1227 \\
\hline & & & & \multicolumn{1}{c}{ Tarefa 2 } \\
Partic. & 1 & 2 & 3 & 4 & 5 & 6 & 7 & 8 & 9 & 10 & Total \\
Freq. & 113 & 151 & 72 & 100 & 179 & 126 & 120 & 89 & 96 & 170 & 1216 \\
\hline & & & & & & Tarefa 3 & & & & & \\
Partic. & 1 & 2 & 3 & 4 & 5 & 6 & 7 & 8 & 9 & 10 & Total \\
Freq. & 75 & 104 & 38 & 105 & 97 & 42 & 96 & 81 & 74 & 76 & 788 \\
\hline
\end{tabular}




\section{Conclusões, limitações e sugestões para futuras pesquisas}

Os efeitos benéficos advindos da utilização de suporte visual para o desempenho de tarefas de produção oral, conforme relatado na literatura (ROBINSON, 1995, em ELLIS, 2003; COHEN, 1994), foram confirmados no presente estudo. O tipo de tarefa e suas características específicas parecem afetar o uso de estratégias de comunicação em termos de frequiência, mas os participantes desta pesquisa tenderam a utilizar consistentemente um determinado grupo de EC para desempenhar as três tarefas. A narrativa baseada em figuras parece ter ocasionado menos problemas com a linguagem, o que resultou na utilização de um menor número de EC. Já as duas narrativas baseadas em tópicos produziram um número bem maior de EC, e o fato de ambas focalizarem tópicos diferentes (memórias de um momento feliz e relato de filme) não levou, em geral, os participantes a recorrer a tipos diferentes de EC.

De qualquer modo, é possível concluir que é necessário ter em mente que tarefas narrativas baseadas em figuras e aquelas baseadas em tópicos afetam a freqüência de uso de EC. Portanto, certo grau de cautela é necessário ao interpretar dados relativos ao uso de EC (e provavelmente de outros aspectos da fala, tais como fluência, complexidade e acurácia) provenientes da utilização de diferentes tipos de tarefas narrativas, tanto em trabalhos de pesquisa quanto em atividades realizadas em sala de aula ou de avaliação.

O presente trabalho contou com um número limitado de participantes, $o$ que impede a generalização dos resultados. As considerações feitas durante a discussão dos resultados se aplicam apenas ao grupo de participantes de nível intermediário investigado neste trabalho. Faz-se necessário replicar esta pesquisa com um número mais expressivo de participantes, de níveis de proficiência variados, para apresentar resultados mais conclusivos sobre o impacto de diferentes tipos de tarefas sobre a utilização de EC. Sugerimos também que outras pesquisas investiguem em que medida os aprendizes têm consciência das EC que utilizam e se elas são eficientes para auxiliá-los a remediar problemas comunicativos.

\section{Nota}

${ }^{1}$ Na taxonomia de Dörnyei e Kormos (1998) umming e erring é uma EC que envolve o uso de expressões de hesitação como er, uh, uhm. 


\section{Referências Bibliográficas}

BACHMAN, L.; PALMER, A. Language testing in practice: Designing and developing useful language tests. Oxford: O.U.P., 1996.

BIALYSTOK, E. Communication strategies: A psychological analysis of secondlanguage use. Oxford: Basil Blackwell, 1990.

BYGATE, M.; SKEHAN, P.; SWAIN, M. Introduction. In: BYGATE, M.; SKEHAN, P.; SWAIN, M. (Eds.). Researching pedagogic tasks: Second language learning, teaching, and testing. London: Longman, 2001, p.1-20.

CHEN, S. Q. A study of communication strategies in interlanguage production by Chinese EFL learners. Language Learning, v. 40, p. 155-87, 1990.

COHEN, A. D. Assessing language ability in the classroom. Boston: Heinle \& Heinle Publishers, 1994.

DÖRNYEI, Z.; KORMOS, J. Problem-solving mechanisms in L2 communication: A psycholinguistic perspective. Studies in Second Language Acquisition, v. 20, p. 349-385, 1998.

DÖRNYEI, Z.; SCOTT, M. L. Communication strategies in a second language: definitions and taxonomies. Language Learning, v. 47, n. 1, p. 173-210, 1997.

ELLIS, R. Task-based language learning and teaching. Oxford University Press, 2003.

FAERCH, C.; KASPER, G. (Eds.). Strategies in interlanguage communication. London: Longman, 1983.

FLYMAN, A. Communication strategies in French as a foreign language. Working Papers, v. 46, p. 57-73, 1997.

FOSTER, P.; SKEHAN, P. The influence of planning and task type on second language performance. Studies in Second Language Acquisition, v. 18, p. 299323, 1996.

KELLERMAN, E. Compensatory strategies in second language research: a critique, a revision, and some (non-) implication for the classroom. In: PHILLIPSON, R.; KELLERMAN, E.; SELINKER, L.; SHARWOOD SMITH, M.; SWAIN, M. (Eds.). Foreign/Second Language Pedagogy Research: a Commemorative Volume for Claus Faerch, 142-61. Clevedon, UK: Multilingual Matters, 1991.

KELLERMAN, E.; BONGAERTS, T.; POULISSE, N. Strategy and system in L2 referential communication. In: ELLIS, R. (Ed.). Second language acquisition in context. Englewood Cliffs, NJ: Prentice Hall, 1987. p. 100-112. 
LEVELT, W. J. M. Speaking: from intention to articulation. Cambridge, MA: MIT Press, 1989.

MANCHÓN, R. M. Fostering the autonomous use of communication strategies in the foreign language classroom. Links \& Letters, v. 7, p. 13-27, 2000.

PARIBAKHT, T. Strategic competence and language proficiency. Applied Linguistics, v. 6, n. 2, p. 132-46, 1985.

POULISSE, N.; SCHILS, E. The influence of task- and proficiency-related factors on the use of compensatory strategies: a quantitative analysis. Language Learning, v. 39, n. 1, p. 15-48, 1989.

PREBIANCA, G. V. V. Communication strategies and L2 speech production. 2004. 318 f. Dissertação (Mestrado em Letras - Inglês) - Universidade Federal de Santa Catarina, Florianópolis.

ROBINSON, P. Task complexity, cognitive resources, and syllabus design: a triadic framework for examining task influences on SLA. In: ROBINSON, P. (Ed.). Cognition and second language instruction. Cambridge: Cambridge University Press, 2001. p. 287-318.

RODRIGUES, C. Estratégias de comunicação em uma língua estrangeira. A perspectiva da sala de aula. Linguagem \& Ensino, v. 2, n. 1, p. 11-35, 1999.

SELINKER, L. Interlanguage. IRAL, v. X, n. 3, p. 209-231, 1972.

SKEHAN, P.; FOSTER, P. The influence of task structure and processing conditions on narrative retellings. Language Learning, v. 49, n. 1, p. 93-120, 1999.

SKEHAN, P. Task-based instruction. Language Teaching, v. 36, p. 1-14, 2003.

TARONE, E. Communication strategies, foreigner talk, and repair in interlanguage. Language Learning, v. 30, p. 417-431, 1980.

VARADI, T. Strategies of target language learner communication: Message adjustment. In: FAERCH, C.; KASPER, G. (Eds.). Strategies in interlanguage communication. London: Longman, 1983. p. 79-99.

YARMOHAMMADI, L.; SEIF, S. More on communication strategies: classification, resources, frequency and underlying processes. IRAL, v. 30, p. 223-232, 1992. 


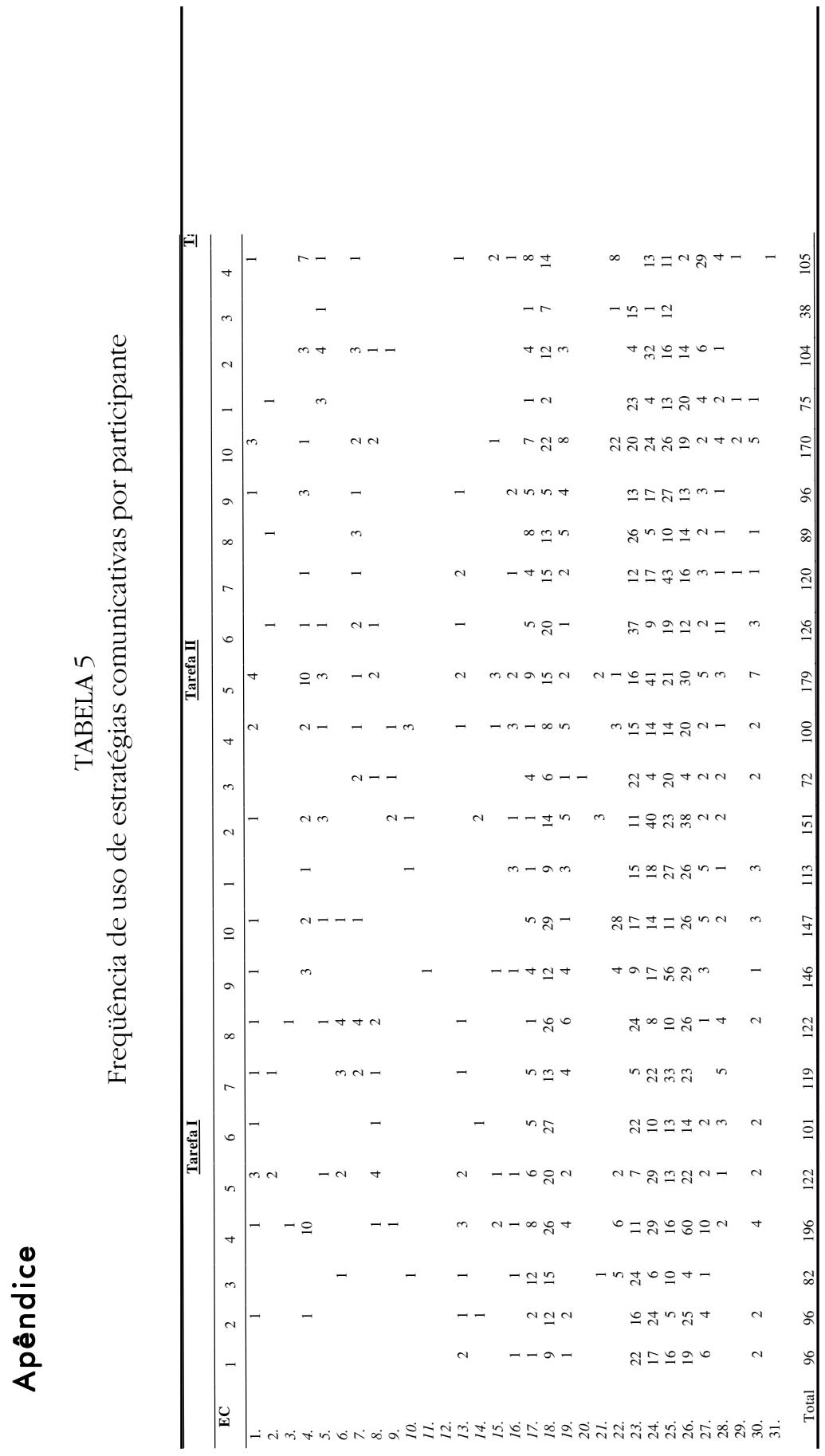

